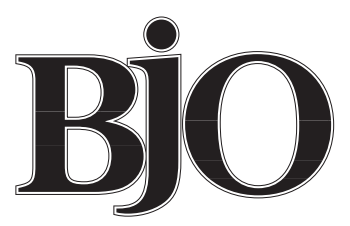

British Journal of Ophthalmology

Even though endophthalmitis is a rare complication of cataract surgery (recent studies report an incidence of $0.12 \%$ in the USA ${ }^{1}$ and $0.17 \%$ in Denmark ${ }^{2}$ ) its treatment continues to be a frustrating problem leading frequently to disastrous visual results. In this issue of the $B \mathcal{F O}(\mathrm{p} 719)$ Okhravi et al assess the effect of a standard treatment protocol on the visual outcome of patients who have been diagnosed as having presumed bacterial endophthalmitis following intraocular surgery or penetrating ocular trauma. This protocol utilises early aggressive treatment with a standard regimen of high dose broad spectrum intraocular and systemic antibiotics. It also assesses the sensitivity of the organisms isolated to the specific antibiotics employed. In spite of aggressive and appropriate broad spectrum antibiotic therapy the final visual outcome was poor, with $55 \%$ of postsurgical and $40 \%$ of post-traumatic cases achieving a final visual acuity of $6 / 60$ or less.

Why is the treatment of endophthalmitis still such an exercise in frustration? Are we somehow missing the point as clinicians, or is intraocular infection a problem in which a successful cure will always remain beyond our grasp? One difficulty is that endophthalmitis is not a single entity but is instead a heterogeneous group of infections with diverse epidemiological presentations and a varying spectrum of pathogenic organisms. In a review of 30 culture positive cases of endophthalmitis in Auckland from 1983 to 1991 , Kent ${ }^{3}$ found 13 early onset postoperative, six delayed onset postoperative, five bleb associated, four post-traumatic, and two endogenous cases. To these five major categories should be added a sixth, infection by contiguous spread. In addition, bleb associated endophthalmitis can be divided into blebitis, which usually has a very favourable visual outcome, and bleb associated vitritis, which has a prognosis as guarded as early onset postoperative endophthalmitis. Endogenous endophthalmitis can be further subclassified into five categories as described by Greenwald et al. ${ }^{4}$ These are anterior focal, posterior focal, anterior diffuse, posterior diffuse, and panophthalmitis. All five categories have different clinical presentations, different visual prognoses, and very specific guidelines for diagnostic evaluation and antibiotic administration.

In addition to the clinical complexity of intraocular infection, every case of endophthalmitis presents the physician with three major issues which profoundly affect visual outcome but over which the clinician has no control.
The first issue is the inherent pathogenicity of the infecting organism. Bacteria which are intrinsically highly pathogenic such as Pseudomonas aeruginosa and Streptococcus pyogenes produce severe intraocular infections that lead invariably to poor visual outcomes. Fortunately, the majority of cases of endophthalmitis are caused by Gram positive bacteria of relatively low pathogenicity. In the Endophthalmitis Vitrectomy Study ${ }^{5}$ Gram positive, coagulase negative staphylococci were the single most common group of isolates representing $70.0 \%$ of all positive cultures from endophthalmitis patients, $94.5 \%$ of whom were postoperative cataract surgery. Speaker et $a l^{6}$ demonstrated by genetic analysis that $82.0 \%$ of coagulase negative staphylococci isolated from intraocular specimens in endophthalmitis are genetically indistinguishable from organisms on the patient's lids. Since virtually all individuals have Staphylococcus epidermidis colonising their eyelids, the source of the infecting organisms during cataract surgery is obvious. It must be emphatically stated, however, that other clinical forms of endophthalmitis are frequently caused by various other pathogens. Alfaro et $a l^{7}$ reported 21 culture positive cases of post-traumatic endophthalmitis in which $25.6 \%$ of the isolates were Staphylococcus species, $20.6 \%$ were Streptococcus species, and $14.7 \%$ were Bacillus species. And Thompson et al ${ }^{8}$ isolated bacilli or staphylococci in $95 \%$ of culture positive cases of endophthalmitis following penetrating injuries with retained intraocular foreign bodies. Likewise, the overwhelming majority of cases of late onset postoperative endophthalmitis are caused by Propionibacterium acnes, ${ }^{9}$ while the pathogens responsible for bleb associated infections correspond with the organisms that produce acute purulent conjunctivitis-Haemophilus influenzae, Streptococcus pneumoniae, and Staphylococcus aureus. Endophthalmitis associated with infection by contiguous spread is most often caused by Pseudomonas species, while the bacteria responsible for endogenous endophthalmitis are a diverse group of organisms that include Bacillus species and Klebsiella pneumoniae as well as multiple streptococcal species and Staphylococcus aureus. ${ }^{10}$

The second major issue affecting visual outcome is the size of the inoculum of the infecting organism. Even a relatively benign pathogen such as Staphylococcus epidermidis can produce an endophthalmitis that is resistant to treatment if a sufficient number of organisms are 
introduced into the vitreous. ${ }^{11}$ All of the techniques for maintaining asepsis in the operating room are directed towards preventing such an event from occurring, but non-postoperative forms of endophthalmitis are exempt from these precautions. The third and perhaps most important issue affecting visual outcome is the time lapse between the onset of symptoms and clinical diagnoses and the initiation of aggressive appropriate therapy. Paradoxically, time is the only factor over which the clinician has a small measure of control. The faintest suspicion that the patient is developing endophthalmitis should lead to the immediate institution of a standardised diagnostic and treatment protocol such as the one described by Okhravi and colleagues in this issue.

Admittedly, there are still questions regarding the standard of therapeutic care. Are systemic antibiotics really necessary for the treatment of endophthalmitis? Has the question of when to do a vitrectomy been definitively answered? In spite of the results of the Endophthalmitis Vitrectomy Study $^{12}$ these grey areas of clinical judgment continue to weigh heavily on clinicians. Perhaps it is the complex nature of endophthalmitis itself that prevents a standard protocol from being devised that can consistently produce a satisfactory visual outcome in this group of patients that are so difficult to treat. Unfortunately, even in the best of hands the results are still frustrating and the ideal treatment for endophthalmitis remains elusive.

JOHN P WHITCHER
Francis I Proctor Foundation for Research in Ophthalmology, Box 0944, University of California San Francisco,

San Francisco, CA 94143-0944, USA

1 Norregaard JC, Thoning H, Bernth-Peterson P, Anderson TF, Javitt JC, Anderson GF. Risk of endophthalmitis after cataract extraction: results from the International Cataract Surgery Outcomes Study. Br 7 Ophthalmol 1997;81:102-6.

2 Javitt JC, Vitale S, Canner JK, Street DA, Krakauer H, McBean AM, et al. National outcomes of cataract extraction. Endophthalmitis following inpatient surgery. Arch Ophthalmol 1991;109:1085-9.

3 Kent DG. Endophthalmitis in Auckland 1983-1991. Aust N Z F Ophthalmol 1993;21:227-36.

4 Greenwald MJ, Wohl LG, Sell CH. Metastatic bacterial endophthalmitis: a contemporary reappraisal. Surv Ophthalmol 1986;31:81-101.

5 Han DP, Wisniewski SR, Wilson LA, Barza M, Vine AK, Doft BH, et al. Spectrum and susceptibilities of microbiologic isolates in the endophthalmitis vitrectomy study. Am f Ophthalmol 1996;122:1-17.

6 Speaker MG, Milch FA, Shah MK, Eisner W, Kreiswirth BN. Role of external bacterial flora in the pathogenesis of acute postoperative endophthalmitis. Ophthalmology 1991;98:639-49.

7 Alfaro DV, Roth D, Liggett PE. Post-traumatic endophthalmitis. Causative organisms, treatment, and prevention. Retina 1994;14:206-11.

8 Thompson JT, Parver LM, Euger CL, Mieler WF, Liggett PE. Infectious endophthalmitis after penetrating injuries with retained intraocular foreign endophthalmitis after penetrating injuries
bodies. Ophthalmology 1993;100:1468-74.

9 Rogers NK, Fox PD, Noble BA, Kerr K, Inglis T. Aggressive management of an epidemic of chronic pseudophakic endophthalmitis: results and literature survey. Br F Ophthalmol 1994;78:115-9.

10 Okada AA, Johnson RP, Liles WC, D'Amico DJ, Baker AS. Endogenous bacterial endoophthalmitis. Report of a ten-year retrospective study. Ophthalmology 1994;101:832-8.

11 Shaarowy A, Grand MG, Meredith TA, Ibanez HE. Persistent endophthalmitis after intravitreal antimicrobial therapy. Ophthalmology 1995;105: 382-7.

12 Endophthalmitis Vitrectomy Study Group. Results of the endophthalmitis vitrectomy study. A randomized trial of immediate vitrectomy and of intravenous antibiotics for the treatment of postoperative bacterial endophintravenous antibiotics for the treatment of pos.
thalmitis. Arch Ophthalmol 1995;113:1479-96.

\section{Oral administration of antigen in the treatment of eye disease}

Feeding antigens to experimental animals can result in oral tolerance, a peripheral immunological non-responsiveness induced by the processing of exogenous antigen through gut associated lymphoid tissue. ${ }^{1}$ Oral administration of autoantigen has been shown to ameliorate disease in a number of rodent models of organ specific autoimmune conditions including experimental autoimmune uveoretinitis (EAU), ${ }^{1-5}$ experimental autoimmune encephalomyelitis (EAE), ${ }^{6}$ experimental granulomatous colitis, ${ }^{7}$ and experimental autoimmune thyroiditis. ${ }^{8}$ Because antigen feeding protocols have been shown to be effective even in sensitised recipients with established disease, induction of oral tolerance represents a novel potential strategy for the treatment of human autoimmune disease, allergy and possibly graft rejection.

The mechanisms involved in induction and maintenance of oral tolerance depend critically upon antigen dosage. ${ }^{13}$ Immunisation with high doses of antigen leads to energy of antigen reactive cells, whereas lower antigen doses induce a form of immune deviation or active suppression. Anergy reflects the situation in which antigen reactive cells are generated but are functionally inactive: the molecular mechanisms involved are uncertain. Immune deviation operates through induction of a population of transforming growth factor beta (TGF- $\beta$ ) secreting regulatory $\mathrm{T}$ cells (Th3 cells) which down regulate immune responses, and by a general skewing towards a Th2 immune response with production of immunomodulatory cytokines IL-4 and IL-10. In most experimental systems, oral tolerance can be augmented by covalent coupling of antigen to the mucosa binding cholera toxin B subunit, which appears to have a strong adjuvant effect, ${ }^{9}$ or by concomitant administration of IL-2. ${ }^{5}$ As with anterior chamber associated immune deviation (ACAID),${ }^{10}$ an intact spleen is necessary for the induction of oral tolerance to ocular antigen. ${ }^{4} \mathrm{~A}$ detailed review of potential pathways to tolerance in autoimmune eye disease has recently been provided by Rizzo and Caspi. ${ }^{11}$

In 1996, Niederkorn and his colleagues demonstrated that oral administration of donor type antigen halved the incidence of murine corneal allograft rejection across multiple major and minor histocompatibility antigen mismatches. ${ }^{12}$ Antigen was administered in the form of tissue cultured, immortalised corneal epithelial and endothelial cells or freshly isolated keratinocytes of donor phenotype. In this issue of the BfO ( $p$ 778), the same group shows that conjugation of cultured corneal cells to the cholera toxin B subunit significantly enhances the efficacy of the regimen in prolonging graft survival, with a single oral dose of antigen reducing the incidence of corneal graft rejection by $36 \%$. Multiple oral doses decrease the incidence of rejection by over $90 \%$. Initiating antigen feeding on the day of corneal transplantation is very effective but oral administration of conjugated corneal cells can be delayed for up to 7 days after corneal transplantation and still produce a significant (albeit lesser) effect. Interestingly, administration of exogenous IL-2 has no augmentative effect but, as expected, recipient splenectomy abolishes induction of oral tolerance.

The majority of reported studies on oral administration of antigen have been performed in rodent models. The question on everyone's lips is, of course, will oral immunisation work in humans? In one clinical trial, patients with multiple sclerosis fed bovine myelin suffered fewer relapses and demonstrated significantly fewer blood borne antigen reactive $\mathrm{T}$ cells ${ }^{13}$ and more $\mathrm{TGF}-\beta$ secreting $\mathrm{T}$ cells ${ }^{14}$ than 